\title{
Philosophiques
}

\section{Jean Grondin, Kant. Avant/après, [Collection La Création de l'Esprit], Paris, Criterion, 1990, 204 pages.}

\section{Mario Dufour}

Volume 19, numéro 1, printemps 1992

URI : https://id.erudit.org/iderudit/027180ar

DOI : https://doi.org/10.7202/027180ar

Aller au sommaire du numéro

Éditeur(s)

Société de philosophie du Québec

ISSN

0316-2923 (imprimé)

1492-1391 (numérique)

Découvrir la revue

Citer ce compte rendu

Dufour, M. (1992). Compte rendu de [Jean Grondin, Kant. Avant/après, [Collection La Création de l'Esprit], Paris, Criterion, 1990, 204 pages.]

Philosophiques, 19(1), 141-144. https://doi.org/10.7202/027180ar d'utilisation que vous pouvez consulter en ligne.

https://apropos.erudit.org/fr/usagers/politique-dutilisation/ 
Jean GRONDIN, Kant. Avant/après, [Collection La Création de l'Esprit], Paris, Criterion, 1990, 204 pages.

par Mario Dufour

Jean Grondin, bien connu pour ses recherches en philosophie allemande, notamment Le tournant dans la pensée de Martin Heidegger (P.U.F., 1987) et Kant et le problème de la philosophie: l'a priori (Vrin, 1989), propose ici un ouvrage d'introduction à la pensée et à l'œuvre de Kant. L'intention d'introduire est menée avec brio par une lecture d'ensemble de l'oeuvre de Kant et de l'entreprise qui s'y dessine: l'ouvrage est clairement organisé, l'exposé est riche, élégant et convaincant, le style précis, les citations remarquablement choisies et interprétées, l'information abondante et la connaissance de l'œuvre de première main. Il offre aux non-initiés une lecture riche et fidèle de l'entreprise de Kant au sein de l'histoire de la philosophie, et aux initiés une réflexion sur la destination de la philosophie qui rappelera l'actualité et le caractère décisif du projet kantien. L'ouvrage s'ordonne en trois chapitres qui respectent une séquence temporelle et historique: avant Kant; Kant; après Kant. Ce découpage permet de saisir l'essentiel de l'entreprise de Kant: contrairement au " préjugé courant " (32) qui fait de Kant le fossoyeur de la métaphysique et l'annonciateur du thème de la "mort de Dieu», il convient de rappeler que l'intention de Kant est de ménager un nouvel avenir à la métaphysique qui passe, selon l'expression de l'auteur, par un " tournant méthodologique " de la raison théorique à la raison pratique et donc par une "réinvention de l'éthique " (99).

Le premier chapitre “ Avant Kant " traite de l'état et du devenir de la philosophie à l'époque de Kant. À l'époque de Kant, l'empirisme et la méthode expérimentale a déjà fait son œuvre et l'émancipation des sciences contraint la métaphysique à une position d'arrière-garde que la philosophie garde encore aujourd'hui. 
La philosophie - entendue comme métaphysique ou science (termes équivalents des Grecs jusqu'à Hegel), recherche rigoureuse du pourquoi, des "principes premiers" (ou derniers), des a priori (universaux et nécessaires) qui transcendent le cadre de notre expérience - perd de son autorité et oscille dans un double-bind: ou bien elle se mue en science expérimentale (empirisme) ou bien elle ressasse des tautologies creuses et abstraites (rationalisme) qu'elle prend pour des réalités (dogmatismes de toutes sortes: de la superstition au matérialisme qui prétend établir que Dieu n'existe pas). L'“ Avant Kant " est marqué par cette "perte de crédibilité de la philosophie" (36) qui se traduira pour le Kant de la Critique de la Raison pure en un double défi à relever: celui de la validité objective de nos concepts (à laquelle l'empirisme et le scepticisme ne peuvent répondre) et celui de savoir si la métaphysique est possible à titre de science (la soif trop humaine d'absolu ne saurait être passion inutile). La notion énigmatique de connaissance synthétique a priori, qui déjoue les partages de l'empirisme et du rationalisme, du synthétique a posteriori et de l'analytique a priori offre le chemin $\mathrm{d}^{\prime}$ une solution à ces deux problèmes et devient alors la question "destinale" de la philosophie (41). On voit bien à quoi cela peut ressembler en physique (tout phénomène a une cause) ou en géométrie (le plus court chemin entre deux points est une ligne droite), mais lorsqu'il s'agit de métaphysique (l'âme est immortelle, il y a une causalité de liberté, Dieu existe) ne sommes-nous pas au-delà des limites raisonnables de l'expérience? On peut constater mais ne peut dissoudre l'impasse métaphysique. Kant s'efforce d'en asseoir la légitimité, à titre de disposition naturelle et inévitable, bien qu'impuissante à constituer effectivement son objet, au sein d'un agir moral qui permet de sauver les visées capitales de la métaphysique spéciale (psychologie, cosmologie, théologie).

Le chapitre "Kant " est, comme il se doit, deux fois plus long que les autres. Dans un cadre restaurateur il brosse un tableau de la philosophie et de l'œuvre de Kant (qui n'est pas un système, 130-132) dans ses aspects épistémologiques, éthiques, historiques et politiques. L'auteur commence bien sûr par une analyse de La Critique de la raison pure. Il extrait avec soins les principales facettes, tout en insistant sur ses obscurités, ressortissant à sa première partie "La théorie transcendantale des éléments de la connaissance pure ": schématisation du donné phénoménal dispersif (phénoménalisme) par les intuitions pures du temps et l'espace (Esthétique trancendantale), et par les structures catégoriales et conceptuelles de l'entendement ressortissant à une logique du vrai basée sur le jugement (Analytique, Première partie de la Logique); exercice naturel de la raison dans le syllogisme qui instancie la tendance d'une remontée du conditionné vers l'inconditionné (la raison dans son usage logique cherche la majeure, la règle générale, la condition universelle) dont traite la Dialectique (Deuxième partie de la Logique) comme logique du faux qui dénonce impitoyablement la subjugation de la 
métaphysique par l'apparence transcendantale et par la prétention de connattre le non-sensible.

Mais il s'agit là, on était prévenu, d'un détour (41), créant, il est vrai, "de toutes pièces l'épistémologie " (42), vers un examen des conditions de possibilité de la métaphysique que Kant relance, non plus du côté des spéculations de la raison pure, mais du côté de "l'efficace " d'un usage légitime d'une raison pratique morale. Si la Dialectique dévoile une raison épuisée, déchirée par l'antinomie entre sa soif d'absolu et son souci de scientificité, la deuxième section de l'ouvrage ("Théorie de la méthode"), et surtout la section "Le Canon", contourne l'impasse métaphysique en un "tournant méthodologique vers la raison pratique ". Selon le Canon, l'usage légitime de la raison pure, "les commentateurs de Kant on eu l'ingénuité de ne pas l'apercevoir ", concerne non pas "l'usage spéculatif mais l'usage pratique de la raison" (97). Le cas de la troisième antinomie, "de loin le plus déterminant" (91), "qui oppose à la causalité mécanique de la nature l'idée d'une causalité par liberté ", apparemment sans issue, distingue des registres bien différents: le phénoménal et l'en soi. L'Idée n'est pas le Concept, ni la pensée, la connaissance.

Il en va d'une métaphysique de la liberté ("clé de voûte, dit la Préface à la Critique de la raison pratique, de tout l'édifice d'un sytème de la raison pure $\%$ ). L'être humain peut s'élever au-dessus de sa condition sensible (animale, égoïste et particularisante) et agir en fonction d'un impératif ou d'une loi ( "qui dit ce qui doit arriver bien que cela peut-être n'arrive jamais ") où chacun est traité comme une fin (liberté pour autrui). L'irruption de la liberté (113), l'indépendance de la volonté, le pouvoir de commencer de soi-même un état (91, 112) relève de la sublimité (Erhabenheit, 115, 130) de l'homme et de sa participation à un monde trans-empirique à même d'influer sur le sensible. La destination morale de l'humanité et l'inscription de la Liberté dans la Nature est invérifiable, mais au moins est-elle suggérée par le progrès dans l'ordre légal ou juridique (146) et présentée, nostalgiquement dans l'Idée du Beau (116-132). Aussi, la rationalité pour un être fini (sans intuition intellectuelle) est-elle nécessairement pratique précaire, ouverte et donc historique. Selon la remarque de Foucault, Kant inaugure au XVIIIe siècle, la première époque à réfléchir sur sa propre historicité, une « ontologie du temps présent" (141), ce qui serait le trait marquant de la modernité (143). L'homme kantien est, dans la suite du cartésianisme, la source ultime de toute légitimation, mais ce que l'homme doit faire de lui-même (se rendre libre et autonome en s'élevant à l'universel) s'actualise non dans une philosophia perennis, mais dans un devenir historique n'autorisant qu'un espoir de finalité rationnelle.

Le dernier chapitre "Après Kant» montre comment l'œuvre de Kant décide de l'avenir de la philosophie. Selon l'auteur, "le projet kantien d'une métaphysique de la nature là vrai dire «laissé 
dans ses tiroirs ", p. 104] et des mœurs remplace, terme pour terme, le doublet classique d'une métaphysique générale (qui soit ontologie) et spéciale " (156). Les premiers héritiers de Kant préoccupés par la question du système ont d'abord essayé de colmater la brèche laissée ouverte entre Nature et Liberté: "l'idée kantienne d'une philosophie cosmique qui ne serait jamais réalisée in concreto n'a pas satisfait l'appétit spéculatif de ses premiers héritiers".

De la législation originaire du Moi de Fichte, en passant par Schelling et la clôture hégelienne, l'absolu prend la finitude pour son contraire, et rend absurde toute morale qui ne soit pas effective: on extrapole le sujet autonome hors des limites que Kant lui assignait. Comme vont en ce sens les développements intéressants que consacre l'auteur à Reinhold (lui-même salué par Kant), à Heidegger (à qui manque au moins deux moments kantiens essentiels à l'éthique: l'exigence d'universalité et l'idée connexe d'une obligation intersubjective) et par suite à Gadamer, Lévinas, Habermas (qui ne saisit pas toute la radicalité de l'impératif catégorique de Kant), etc., la réflexion philosophique post-kantienne, avec les hégéliens de gauche (Marx, Kieggerard, Schopenhauer, voire Nietzsche), est orientée par la reprise de la métaphysique spéciale et conduit à la réhabilitation de la philosophie pratique. Elle est une "éthique, le plus souvent greffée sur une philosophie de l'histoire " (156). D’autre part, le phénoménalisme selon lequel on ne connaît que les objets de l'expérience, (une tautologie, mais seulement depuis Kant, 134) qui ruine l'ontologie traditionnelle (au moins jusqu'à Heidegger, 154), et qui conduit au programme plus modeste d'une analytique de l'entendement pur se contentant de formuler les conditions de possibilité de l'expérience et de sa pénétration scientifique, le phénoménalisme, donc, a pour legs les théories des sciences et l'épistémologie: positivisme néo-kantien (175), sciences humaines (Geisteswissenchaften, Dilthey, 176), Popper (177), analyse du langage comme a priori de toute expérience, voire phénoménologie transcendantale de Husserl (179-180), et plus curieusement le déconstructivisme et les penseurs post-modernes qui poursuivraient l'entreprise de critique kantienne de la métaphysique mais sans se soucier du tournant kantien vers la raison pratique $(53,190)$.

On peut ne pas partager toutes les directions que cet ouvrage propose. Mais il provoque des rapprochements stimulants qui mettent au jour toute la richesse et l'historicité de l'œuvre de Kant, et, surtout, le sauvetage des visées capitales de la métaphysique spéciale (immortalité de l'âme, liberté, Dieu) par la cohérence interne de sa philosophie pratique.

Département de philosophie Université du Québec à Montréal 